\title{
Land heterogeneity stimulates intake rate during grazing trips
}

\author{
M Meuret ${ }^{1}, \mathrm{C}$ Viaux ${ }^{1}, \mathrm{~J}$ Chadœuf ${ }^{2}$ \\ 1 INRA-SAD-Écodéveloppement; \\ 2 INRA-Biométrie, domaine Saint-Paul, 84143 Montfavet cedex, France
}

Shepherds say that a meal is organised during a trip so as to divide different resources into a temporal order that stimulates the appetite. We have attempted to assess the magnitude of the stimulation induced by changes of vegetation types, whether or not they are related to direct intervention by the shepherd.

Massive ingestion in two $3 \mathrm{~h}$ meals within a Quercus pubescens Wild coppice at daily intervals of $10 \mathrm{~h}$, supplemented by $450 \mathrm{~g}$ of barley and minerals, allowed a milk production of $2.5 \mathrm{l} / \mathrm{d} 6$ months after kidding (Meuret, 1989). Mapping at $1: 2000$ of the 110 ha of grazing land identified 605 homogeneous land units, according to $5 \mathrm{cri}-$ teria organised into $(n)$ classes: nature and structure of the vegetation $(n=7)$; penetrability (4); shrub cover (4); forage abundance (4); and forage consumption rate (4). A $k$-means clustering by Leas grouped the units into 5 synthetic classes. After 4 days' acclimatisation, a sample goat was continuously monitored by 2 observers during 10 consecutive meals. The grazing location, movements and ingestion (divided into intake units) were recorded continuously by direct observation (Meuret, 1989) and the data was grouped for each minute. Visible and audible interactions with the shepherd were noted. A 3rd observer recorded the activity pattern of the herd at 20min intervals by scanning all the 41 animals.

The intake rate at time $(t)$ ( $g$ dry matter ingested (DMI)/minekg BW0.75) was not correlated with any of the individual environmental criteria. Auto correlations carried out on time series showed that intake is correlated with individual movements. Ingestion kinetics were analysed by identifying sequences of accelerated ingestion within each meal. After smoothing of the intake by the $k_{\text {smooth }}$ function of Splus, an acceleration was defined as follows: after a period $\geq 3 \mathrm{~min}$ at less than $0.23 \mathrm{~g}$, the intake must reach a minimum of $0.46 \mathrm{~g}$ in less than 20 min and must remain greater than $0.37 \mathrm{~g}$ for at least $5 \mathrm{~min}$. Ten meals of $171 \pm 11$ min contained 41 accelerations, which accounted for $37 \%$ of the time and $58.8 \%$ of the total ingestion. They usually involved several ingested plant species. Accelerations were distributed as follows: start of the trip (10\%); without direct intervention by the shepherd $(48 \%)$; and with intervention (42\%). Without intervention, half were recorded during changes in synthetic land units, preceded by movements of the entire herd in $7 \%$ of cases and without in $17 \%$. Interventions were followed by an acceleration almost only when they provoked a change in unit, preceded by movements of the herd in $25 \%$ of cases and without in $12 \%$. The land heterogeneity is used to stimulate intake by increasing the possible food choice during the grazing trip. Intake is then more dynamic and higher $( \pm 60 \mathrm{gDM} / \mathrm{kg}$ BWo.75/meal) than in cows whose green fodder is renewed in the trough during the meal (Suzuki et al, 1973).

Meuret M (1989) XVIth Intern Grassld Cong, 4-11 March, Nice, France, 941-942

Suzuki S, Chine Y, Hidari H (1973) J Zootech Sci $44,181-187$ 